\title{
Beyond Accidents: A Back-Analysis on Conveyor Belt Injury for a Better Design for Maintenance Operations
}

\author{
${ }^{1}$ Alberto Martinetti, ${ }^{1}$ Leo A.M. van Dongen and ${ }^{2}$ Raffaele Romano \\ ${ }^{I}$ Design, Production and Management, University of Twente, The Netherlands \\ ${ }^{2}$ Mining Office, Turin Province Office, Italy
}

Article history

Received: 9-11-2016

Revised: 27-12-2016

Accepted: 29-12-2016

Corresponding Author: Alberto Martinetti

Design, Production and Management, University of

Twente, The Netherlands

Email: a.martinetti@utwente.nl

\begin{abstract}
The conveyor belt represents one of the most commonly used methods of transporting bulk materials today. As highlighted multiple times in research, the moving parts are a critical issue causing accidents and injuries and several of these are strictly related to the opportunity to enter in working zones during maintenance activities, considered high-risk activity due to the nature of the job. For these reasons, maintenance operations have to be carefully planned during the design phase, with the adoption of appropriate safety measures for the maintenance crew and other people present in the area. On the other hand, preventative measures have to be put in action for maintenance operations and daily work. Analysing the accident reports related to the crushing and milling plants obtained from the several data bases and identifying with a Cause-Consequences analysis the root causes of an injury occurred in an Italian quarry, the paper underlines critical issues of the conveyor belts during maintenance operations, identifies common errors in the system management that affect the workers' safety and proposes an approach in order to improve the maintainability and the safety during the design phase.
\end{abstract}

Keywords: Design for Maintenance Operations, Injuries, Conveyor Belt, Cause-Consequences Analysis, Safety, Mining Industry

\section{Introduction}

For the mining industry, the application of conveyor belts holds a vital role for transferring the bulk materials from the production point to the processing till to the storing point. Despite the significant reductions in accidents over the last two decades, the number of fatalities and injuries related to moving parts (Chinniah, 2015 ) and to the conveyor belt remains remarkably high (Groves et al., 2007). According to the data provided by Ruff et al. (2011) related to the U.S. mining industry the haul trucks, conveyors and front-end-loaders subcategories account for more than $40 \%$ of the fatalities and the accidents attributable to the conveyor represent $10 \%$ of all fatalities occurred in the last decade in the mining industry. In addition, even when the injuries are not serious, they result in lost work time; over a fiveyear period (2006-2010), the average number of lostwork days per injury was 35 . Aside of ethical and moral considerations, it means a significant production efficiency reduction and not-negligible costs for time lost, sanctions, medical expenses, unemployment insurance, extra working hours, marketing and brandrelated actions, insurances, less individual productivity, higher turn-over, internal social problems and legal assistance.

The crushing and milling plants and ancillary structures are a crucial equipment in meeting production requirements while keeping capital and operational costs to a minimum. But, to ensure these achievements several principal design parameters that drive crushing plant selection and configuration have to be taken into account: production requirements, capital cost, ore characteristics, project location, life of mine/expansion plans, climatic conditions, maintenance and safety requirements. The modern plant includes safety guards around all moving equipment and emergency pull-cords on both sides of any conveyors with personnel access. But, the application of safety measures could only produce a risk reduction of $65-75 \%$ (Bagherpour et al., 2015).

Moreover, a good design approach should include also maintenance operations as starting parameters to ensure that the task is performed without having to remove parts. Unfortunately, most of the times during maintenance operations the effectiveness of the safety guards is not enough to prevent injuries and human errors. Therefore, one of the main challenge is to improve these non-standard working conditions. 


\section{Accidents statistic in Italian crushing and milling plants 2008 - 2012}

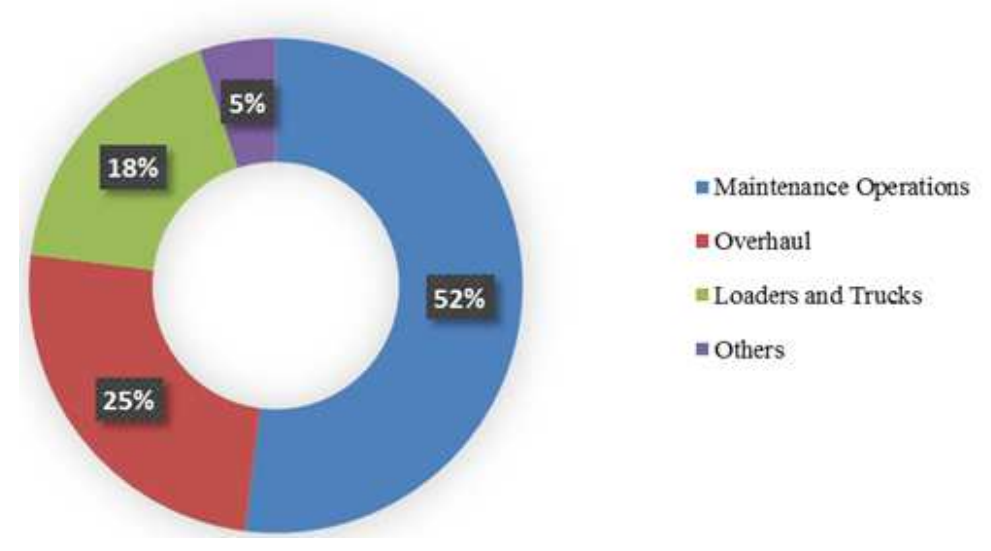

Fig. 1. Accidents statistic in Italian crushing and milling plants (2008 - 2012)

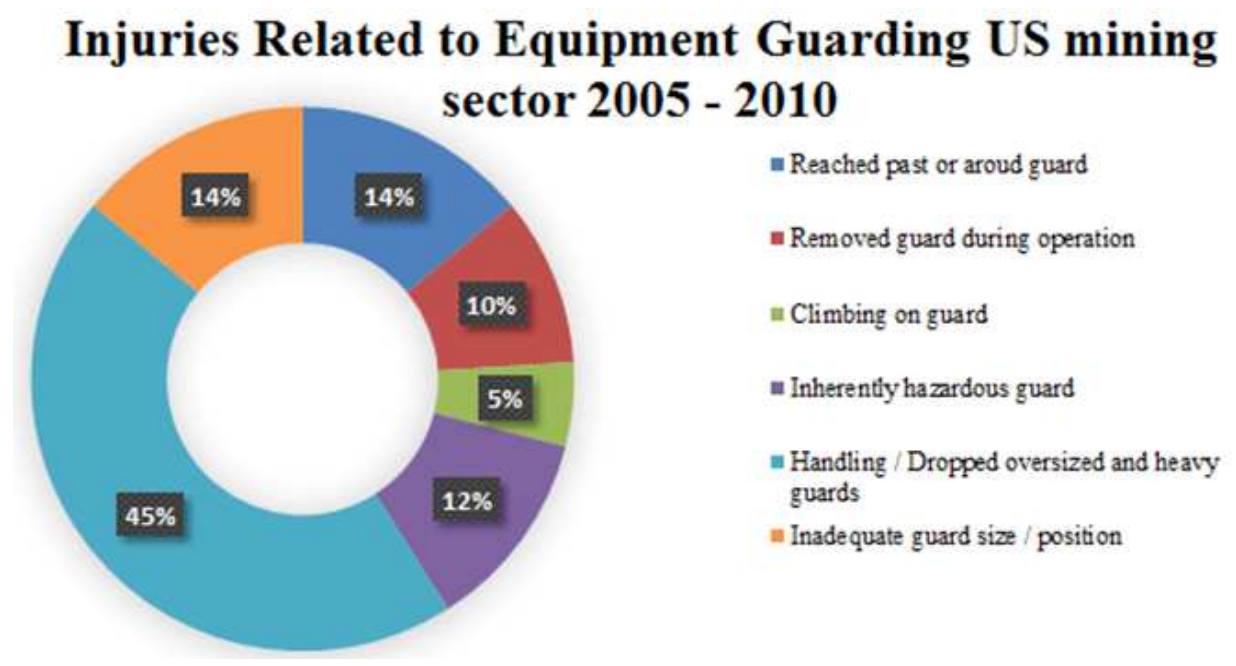

Fig. 2. Injuries Related to Equipment Guarding in US mining sector (2005 - 2010)

How to avoid these unsafe situations taking into account the technical requirements and needs of the equipment? At which level the preventive actions have to be taken? The paper tries to pinpoint the difficult.

\section{The Maintenance Operations: an Activity with Additional Risks}

Maintenance work includes different operations and activities performed often in harsh and difficult conditions. As described by the European Standard EN 13306 (2010), maintenance concerns the "combination of all technical, administrative and managerial actions during the life cycle of an item intended to retain it in, or restore it to, a state in which it can perform the required function".

Requiring multiple skills to the technicians for covering different tasks, maintenance operations increase the risks associated to every working environment due to strict schedules and unplanned activities often are required involving a number of specific hazards, such as disassembly and reassembly with the related risks (Väyrynen et al., 2014). These factors represent indeed a relevant degradation in terms of safety and health for the technicians.

As discussed also by Luzzi et al. (2013) the European statistic shows a serious degradation of the safety conditions and a not negligible increase of fatalities 10$15 \%$ (European Agency for Safety and Health at Work, 2010) among workers involved in maintenance activities.

In Finland, the most common causes for accidents in maintenance operations were crushing and falling in the period 1994-2004. Crushing was reported as the accident cause in $40 \%$ of severe accidents and in almost $30 \%$ of fatal accidents (Lind and Nenonen, 2008). Different 
factors and various kinds of deficiencies contributed to the accidents, among which were working while a machine is in motion and dangerous working practices. Most deficiencies were related with machinery safety devices, work guidance, risk assessment and human behaviour.

Sbarzaglia and Correggia (2012) underline the criticalities related to the maintenance operations. In the Italian mining sector in the period 2008-2012, maintenance operations in crushing and milling plants were identified as the accident situation in $52 \%$ (Fig. 1) of all cases: the highest-risk operations in the mining activities.

Vatn and Aven (2010) clearly highlighted how the injuries related to maintenance jobs born always in human errors, in the design and management of the space and in organizational factors (management and supervision).

Statistical Phenomena of the Conveyor Belt Accidents: Different Countries, same Issues

As also proved by the reports on fatal and serious conveyor belt related injuries in the manufacturing and processing sector obtained from the Mine Safety and Health Administration (MSHA), a large amount of accidents can be attributed to accessibility to danger zones.

The data available from 2005 to 2010 (MSHA, 2010) for the US mining sector (Fig. 2) show the most common situations related to the equipment guarding. In the analysed period, the MSHA standards on moving machine parts (MSHA 30 CFR $\S 56 / 57.14107$ ) were violated more than 35,000 times.

A collection of more than 80 fatalities related to conveyor belts occurred in Canada shows that more than $55 \%$ of total accidents involves the pulleys and other rolling parts of the system (OSHC Quebec, 2003) and the majority of these happened during maintenance activities (sharpening blades, lubricating, adjusting, installing guards, repairing). On one hand it becomes evident that it is vital to improve and implement preventative measures in order to provide the right safety standards to the personnel that is working near this area with more extensive design-out for maintenance; on the other hand, it is mandatory to restore the right level of attention and the proper risk perception of the workers with frequent information and training activities. Moreover, as general approach well discussed by Tappura et al. (2014) and highlighted by the OHSAS 18001:2007 standard (2007), the production and industry management covers a fundamental role for improving the OHS issues in terms of organizational supports strategic decision. And this paradigm becomes even more true and necessary in the prevention of injuries and fatalities in SMEs due to a large and recognized difficulty in allocating resources in OHS topics (Hasle et al., 2009).

\section{An Occupational Injury Occurred on Conveyor Belt during the Maintenance Operations in a SME}

As discussed in the previous paragraphs and underlined by Blaise et al. (2014), OHS issue of the technicians involved in repairing and restoring systems lies on multiple hazards not always predictable. Moreover, Bonafede et al. (2016), highlighted clearly that the OHS training among the SMEs is even more difficult due to the lack of resources and time creating situations difficult to manage with limited knowledge of the related hazards.

To better understand the explained issue, an injury occurred in the first hours of the working shift during the maintenance operations of a conveyor belt in an Italian crushing and milling plant for sorting sand and gravel is presented and analyzed. The data available and the gathered information were obtained by the mining inspectors during the investigation required by the Prosecutor's Office to establish the root causes and the possible preventive actions that would have avoided the injury. A brief description of a common conveyor belt is also provided in order to pinpoint the main technical characteristics and hazards to connect to possible OHS issues during the analysis.

\section{Conveyor Belt: Components, Maintenance and Safety Guards}

To clearly understand the hazards and safety issues involved in the analysed machinery, it is necessary to define the main components of a conveyor belt (Table 1) and its general layout (Fig. 3).

A conveyor belt system mainly consists of two or more pulleys with an endless loop; according to the type of conveyor belt, one or more pulleys are powered (drive/head pulleys) and provide the movement to the belt and to the material on the belt forward.

As every machine, the conveyor belt requires different types of maintenance operations and inspections to keep every part reliable ensuring safety and efficiency of the system during all the life span. Daily, weekly, monthly, semi-annually maintenance activities have to be performed for cleaning conveyor, examining and replacing pulleys, bearing, belts, rollers, electrical cables and mounting points and changing fluid in gearboxes.

From the safety prospective, in and around conveyor belts usually several hazards related to moving parts are present. The regulations (European Directive 2006/42) and the A-B-C type standards (EN ISO 12100:2010, EN $953: 1997+\mathrm{A} 1: 2009$, EN $741: 2000+\mathrm{A} 1: 2010)$ on conveyor belt impose to the manufacturers to design and built safe machineries and to the end users to maintain the equipment as suggested in the product's manual. To reduce the risk exposure of the workers, preventive measures must be taken place intrinsic safety principles. 


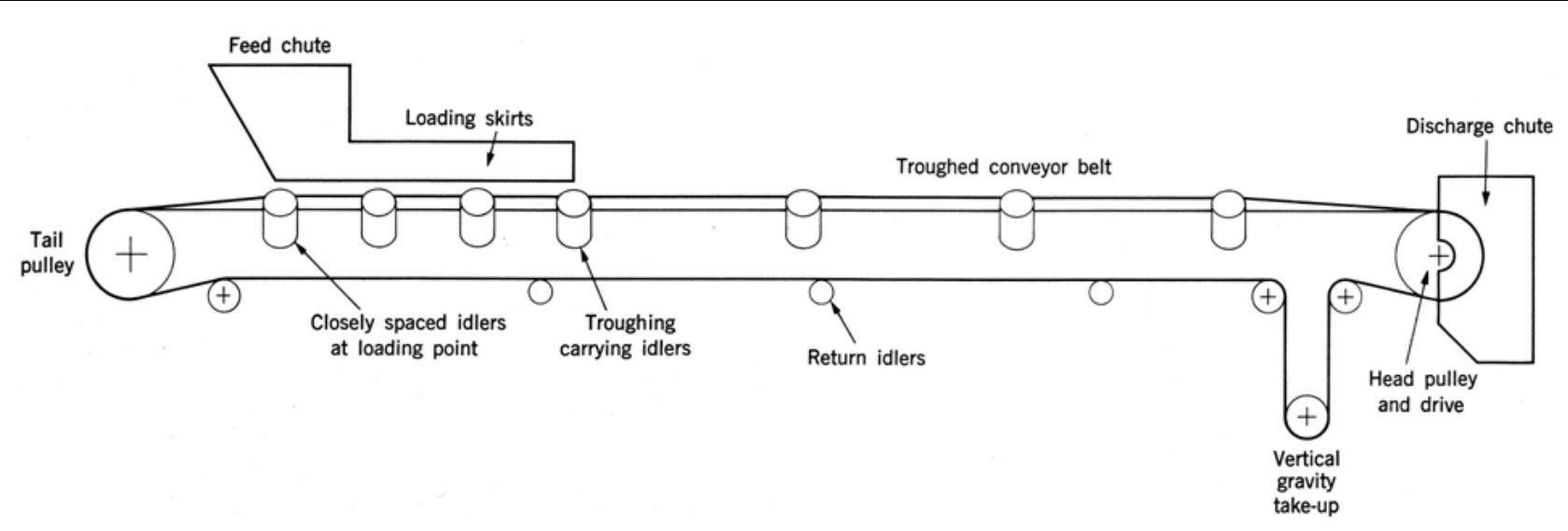

Fig. 3. Scheme of a conveyor belt

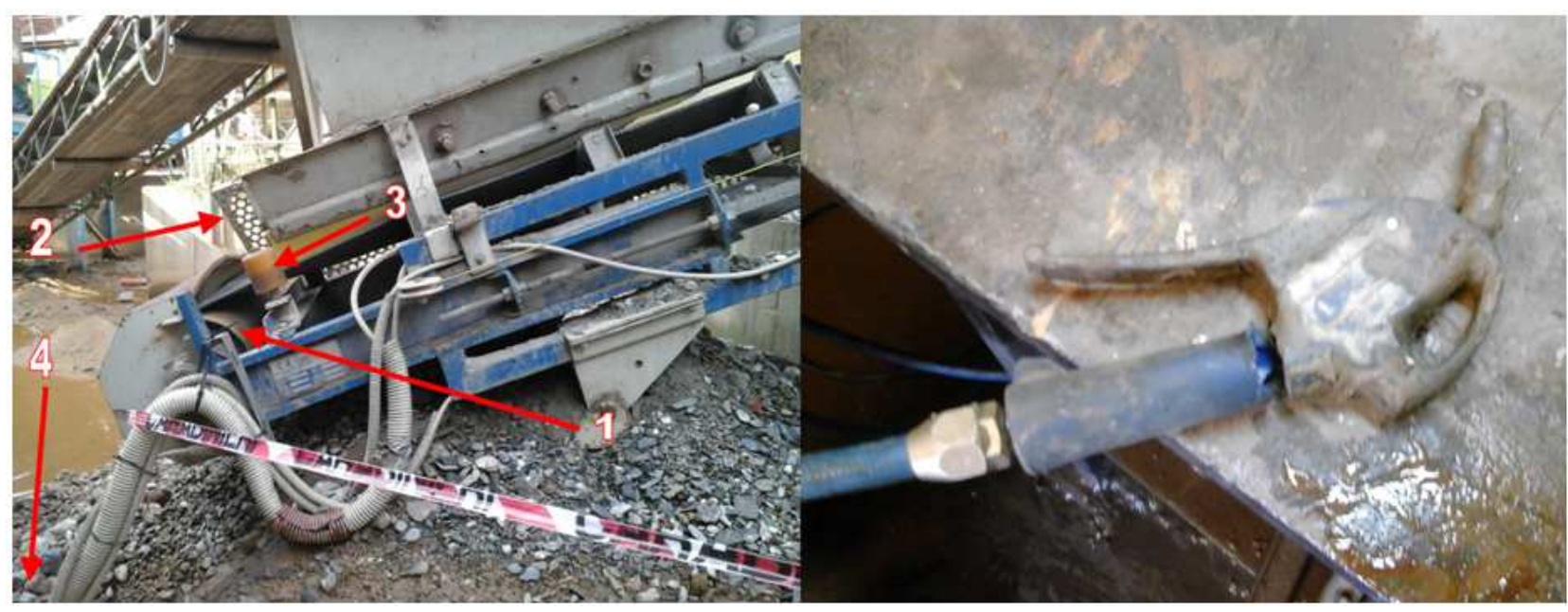

Fig. 4. Left picture. Section of the conveyor belt involved in the injury as observed by the investigators: 1 . Tail pulley of the conveyor belt; 2 . Side fixed guard mounted only on the left side of the conveyor belt; 3 . Load carrying roller replaced during the operation; 4. Position of the air gun after the accident. Right picture: air gun involved in the accident

Table 1. Conveyor belt components

\begin{tabular}{|c|c|}
\hline Conveyor belt component & Function \\
\hline Troughed Conveyor Belt & To convey or transport material \\
\hline Troughing Load Carrying idlers/rollers & Helping the conveyor in carrying the load \\
\hline Return idlers/rollers & Helping the belt in minimizing the friction \\
\hline Vertical gravity take-up & Ensuring proper belt tension \\
\hline Head pulley and drive mechanism & Transferring the movement to the conveyor belt \\
\hline Tail pulley & Bearing the conveyor belt \\
\hline Feed chute and loading skirts & Feeding properly the conveyor belt \\
\hline Discharge chute & Normalizing the material out of the belt \\
\hline Closely spaced idlers/rollers at the loading point & Creating the proper shape of the belt to receive the material \\
\hline Table 2. Conveyor belt safety $\varepsilon$ & \\
\hline Conveyor belt safety guards & Characteristics \\
\hline Fixed guards & Usually removable with special tools \\
\hline Surrounding fixed guards & Completely or partially surround the danger zone \\
\hline Barrier guards & Prevent the access to danger zones \\
\hline Fixed in-running nip guards & Placed at a height of an in-running nip that will not allow access to this zone \\
\hline Interlocked guards with guard locking & Will stop the machinery if the operator will open the gate. \\
\hline
\end{tabular}


In the Table 2 the two type of safety guards used in the conveyor belt are described.

\section{Dynamics of the Injury and the Involved Operations}

At the moment of the accident two employees were working in the crushing and milling plant of small Italian enterprise to replace a load carrying roller of the conveyor belt (Fig. 4). According to the witnesses, one of the workers stopped the machine, disconnected the machine from the electric grid (as suggested by the manual of the machinery) and removed the two side fixed guards (by using a specific tool) to start the maintenance operations.

After the replacement of the load carrying roller, the employee decided to test the conveyor belt. But, he remounted only one of the fixed guards in order to, as reported by the worker himself, save time for further adjustments after the restart of the machinery.

The employee went to the operations cabin to reconnect all the machine's sources of power and to restart the conveyor belt; to perform a further cleaning on the conveyor belt tail pulley with a compressed air gun, he entered in the danger zone while the conveyor belt was working. It is highly likely that during the cleaning operations the compressed air gun was caught in the nip point of the pulley where the conveyor belt ran. The tail pulled his hand and arm into the nip point, causing the multiple laceration. After the accident, the employee was hospitalized and he suffered the amputation of his right arm.

\section{Why to Analyze the Injury: An Opportunity to Understand the Root Causes}

A back analysis of the incident is always highly recommended (in this case required by the prosecutor's office) to underline the root causes leading to the accident, to highlight the critical issues of the conveyor belt in relation with the human behaviour and technical measures suggesting solutions as well to improve the workers' safety. But, to investigate the accident and to identify the root causes, it is necessary to carefully choose the correct Hazard evaluation technique.

\section{The Accident Analysis Technique: A Post-Event Investigation Protocol based on Cause- Consequence Technique}

Accident analysis techniques should provide appropriate input to others investigating complementary aspects of the system (Kontogiannis et al., 2000), combining different methodologies to better understand the problem in terms of human or casual factors. One of the most common mistakes during a risk analysis process is starting to consider the human behaviour as root cause for failures, violations and unsafe actions (even though most of the times it is the easiest and fast solution to identify the problem). The European Directives in the field of Health and Safety (Council Directive 89/391 EEC) and the technical literature (Reason, 2000) require as first action to implement managerial level solutions to reduce human errors. As rightly stated by Anderson (1995) on the tunnelling safety: "to consider as cause of the accident the unsafe or incorrect behaviour of the worker does not appear a good idea anymore".

Consequently, to equally take into account during the investigation the human factor, the work organization and the technical safety measures of the system, a postevent technique based on a combination of CauseConsequence and Fault Tree Analysis (Demichela et al., 2011) is utilized in the paper in order to minimize the incompleteness and subjectivity of the analyst. The chosen technique formally implements the tree-structure of Fault tree analysis, giving the opportunity to develop symmetrical investigation paths for identifying the event causes and the more suitable corrective actions.

According to the characteristics of the mentioned Hazard Identification techniques embedded in the analysis (Center for Chemical Process Safety of the American Institute of Chemical Engineers, 1992), the application of this post-event investigation protocol permits (i) to identify the $1 \mathrm{st}$ and 2 nd intermediate causes drawing uniquely the chain events of the accident, (ii) to find the correct position of the root causes in the process of Risk analysis (iii) and to evaluate suitable corrective actions. The Table 3 summarizes the cause categories, the relation with the event and the database used to create the model; the identified cause categories represent the different step of the protocol and will be pinpoint during the application on the analyzed accident. As described by the authors of the post-event protocol, the European Statistics on Accidents at Work (ESAW) and the detailed information gathered from extensive case histories of injuries carried out within Prosecutor investigations were the foundations for the development and the validation of the method.

\section{The Results of the Analysis on the Accident}

Reason (2000) describes how safety levels drive the methodology to prevent failures, accidents and human errors. Moreover, a correct risk assessment and management has to be considered as primary approach for the OHS the improvements of the working conditions under which humans work instead of focusing to change the human behaviour. From these considerations every reliable risk analysis should start. To put in evidence the strict connection between human behaviour, technical measures and the risk management, the analysis sets three different questions to understand which safety levels failed during the accident:

- How the safety levels of the system perform

- Why the safety levels failed

- How the safety levels affected the accident 


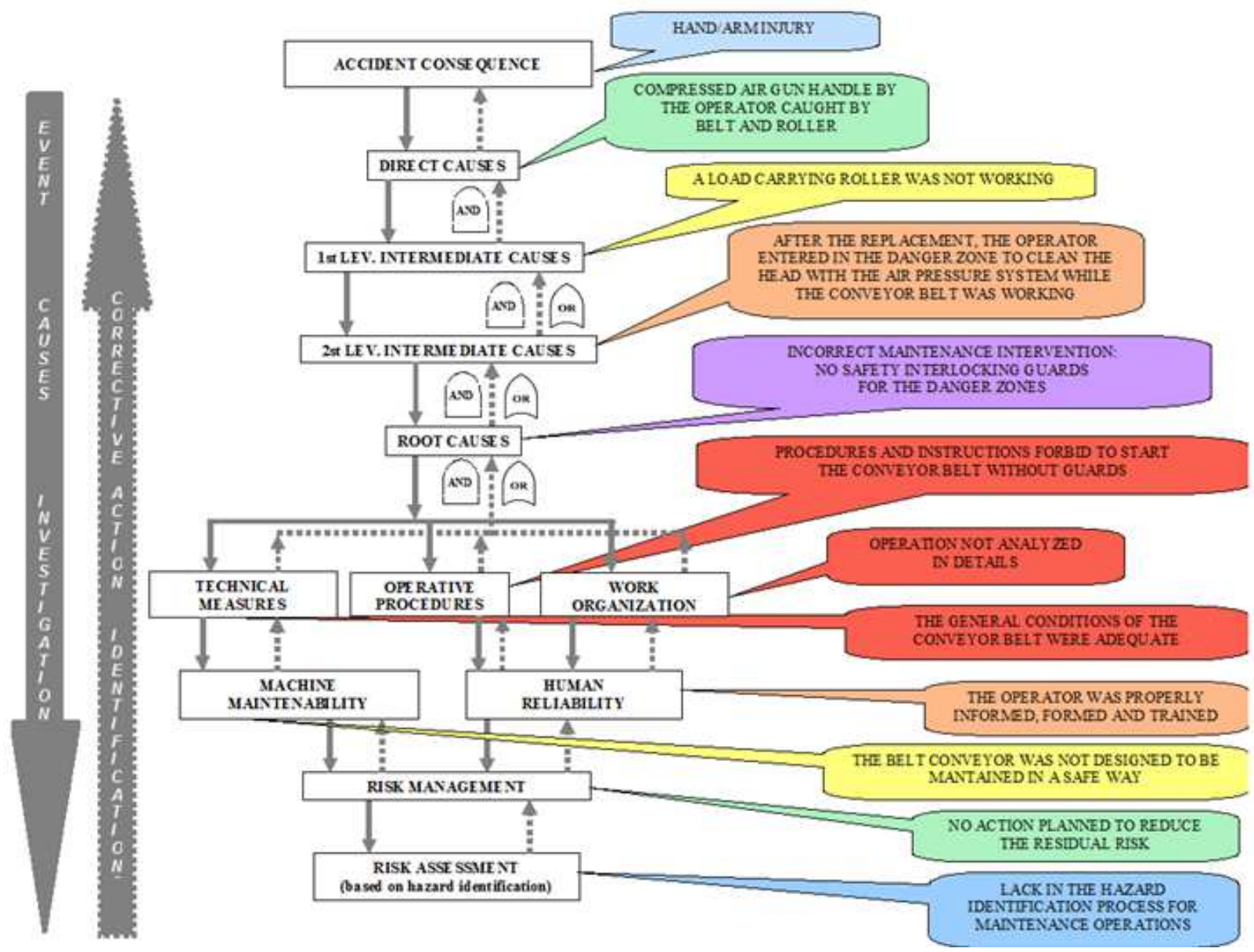

Fig. 5. Post-event investigation protocol applied to the accident occurred on the belt conveyor during maintenance operations

Table 3. Cause categories, events and database event

\begin{tabular}{|c|c|c|}
\hline Cause categories & Event & Database event \\
\hline Direct cause & $\begin{array}{l}\text { Event directly connected to the } \\
\text { accident consequences }\end{array}$ & ESAW database - Type of Injury \\
\hline $1^{\text {st }}$ intermediate cause & Event related to the direct cause & ESAW database - Deviations \\
\hline $2^{\text {nd }}$ intermediate cause & Event directly connected to the root cause & Obtained by the criticalities related to the root causes \\
\hline Root cause & The top event & $\begin{array}{l}\text { Detailed information acquired from a robust } \\
\text { case history of occupational } \\
\text { injuries analyzed by the authors of the protocol }\end{array}$ \\
\hline $\begin{array}{l}\text { Macro categories to pool the } \\
\text { root causes }\end{array}$ & $\begin{array}{l}\text { Technical, operative and work } \\
\text { organization failure }\end{array}$ & $\begin{array}{l}\text { Gathered from the occupational risk } \\
\text { management standards }\end{array}$ \\
\hline
\end{tabular}

Table 4. How the safety levels performed, failed and affected the accident

\begin{tabular}{|c|c|c|c|}
\hline Safety Level & How the safety level performed & Why the safety level failed & How the safety level affected the accident \\
\hline Fixed guard & Failed & The fixed guard was not replaced & $\begin{array}{l}\text { Did not avoid the contact between the employee and } \\
\text { the machine part }\end{array}$ \\
\hline Interlocking guard & Not applicable & $\begin{array}{l}\text { The interlock guard was not } \\
\text { installed on the belt conveyor }\end{array}$ & $\begin{array}{l}\text { Did not avoid the entrance of the employee } \\
\text { in the danger zone }\end{array}$ \\
\hline $\begin{array}{l}\text { Employee formation } \\
\text { and training }\end{array}$ & Failed & $\begin{array}{l}\text { The formation and training cannot } \\
\text { change the human behaviour }\end{array}$ & $\begin{array}{l}\text { The training course did not help the employee to } \\
\text { restore the proper level of attention on the possible risks }\end{array}$ \\
\hline Risk Management & Failed & $\begin{array}{l}\text { Maintenance operations were } \\
\text { often carried on under time pressure }\end{array}$ & $\begin{array}{l}\text { The risk management did not avoid possible human } \\
\text { errors during maintenance under specific } \\
\text { working conditions }\end{array}$ \\
\hline
\end{tabular}


In the Table 4 are listed the identified safety levels involved in the accident, how they performed and why they failed.

The causes' identification offered by the chosen investigation methodology pinpoints (Fig. 5) how the responsibilities (root causes) of the accident should be addressed both (i) to a lack in the maintainability of the belt conveyor, (ii) to a risk underestimation of the maintenance operations during the risk assessment and (iii) to the negligence of the employee who decided to restart the conveyor belt without mounting all the side fixed guards. Such a risk underestimation of the possible hazards during the replacement of a loading carrying roller let to the employee to get in contact with the tail pulley with the compressed air gun.

\section{A Design for Safe Maintenance Operations Decision Process for Minimizing the Risk}

As highlighted by the analysis of the accident, a lack in the maintainability of the belt conveyor increases the risk for the workers during the maintenance operations and the Mean Time To Repair (MTTR), defined as average time required to repair a system, affecting the availability of the machine. Horberry et al. (2010), underlining how the continuous growth of new technologies such as remote control, vision enhancement technologies, continuous haulage and automated equipment in the mining sector generates potential for new health and safety risks, recognizes in the design stage the best moment to minimize these issues and to make more robust the accident prevention. The usercentered design perspective should be able to combine the automation systems with all the human elements (Lynas and Horberry, 2011).

Starting from these considerations and taking into account a development strategy for addressing reliability, maintainability and safety in systems engineering process proposed by Blanchard and Fabrycky (2014) and the interesting guidelines identified by Mulder et al. (2012) and underlined by Vanaker and van Diepen (2016), a Design for Safe Maintenance Operations Decision Process is created (Fig. 6) and discussed in paragraphs 4.1, 4.2, 4.3 and 4.4. The aim of the designed process is to pinpoint the most important information to gather, analyse and include in the design of a mining equipment, as the belt conveyor, in order to reach reliability, maintainability and safety for all the lifespan providing a basic engineering pillars and general solutions about design decisions.

\section{Structure of the Process: Blocks and Phases}

The design process is based on six different phases borrowed from Blanchard's approach (2014) as shown in Table 5, that could be considered as the key decision points for an effective design of an equipment. During all the decision process a symmetry among the phases is maintained to pinpoint a clear interaction between the reliability, maintainability and safety topics that will be considered as an unique element.

In the flow chart, 5 different block types that represent an action to take or a moment during the design process are introduced: start of the diagram block, process block (to describe a set of tasks to perform), a checking block (to check if the right information were taken into account in the steps before), a decision block (to take a decision) and the end of the diagram block. The different block are linked through flow connectors (solid lines) that drive the process; in addition to the these, there are special connectors (dotted lines) that represent both feedback information flows to gather important data during test, production and operating moments and information interactions among the developers and analysts to integrate the different aspects in the best and effective way.

\section{Working Group, Data Analysis and Requirements Definition: Phase I, II, III}

The process starts with the definition of the working group (Phase I); equipment developer, maintenance developer and safety analyst are essential for tackling the issues highlighted in the analysis of the accident in order to create a perfect match in terms of technical performance and risk reduction. It is vital that the introduction of the three experts in the design is simultaneous for developing right from the beginning all the aspects related to reliability, maintainability and safety.

The second step (Phase II) is the gathering and the analysis of all the historical data: components, parts and sub-systems reliability and failures to reduce the downtime of the equipment, MTTR and easiness to maintain the equipment in order to find out which configuration has to be improved for human-machine interaction and the accidents related to the equipment to identify which are the most common and dangerous hazards not yet solved to reduce in the design stage. Due to the relevance of this step, a checking block placed down line is a mandatory to verify if enough and proper data was collected.

In the Phase III the working group collects and checks customer requirements, technical requirements, regulations and laws requirements and functional safety approaches; as highlighted by Blaise et al. (2003), the integration of user expectations and the interactions between them and the design process helps to reduce the number of potential errors during the project of the equipment. Moreover, a clear picture of the conditions and environments in which the product will be used by the customer is required to assess also the possible unexpected events related to external factors (climate factors, production site layout, possible interactions with other machines) not directly related to the product itself or to the user. 


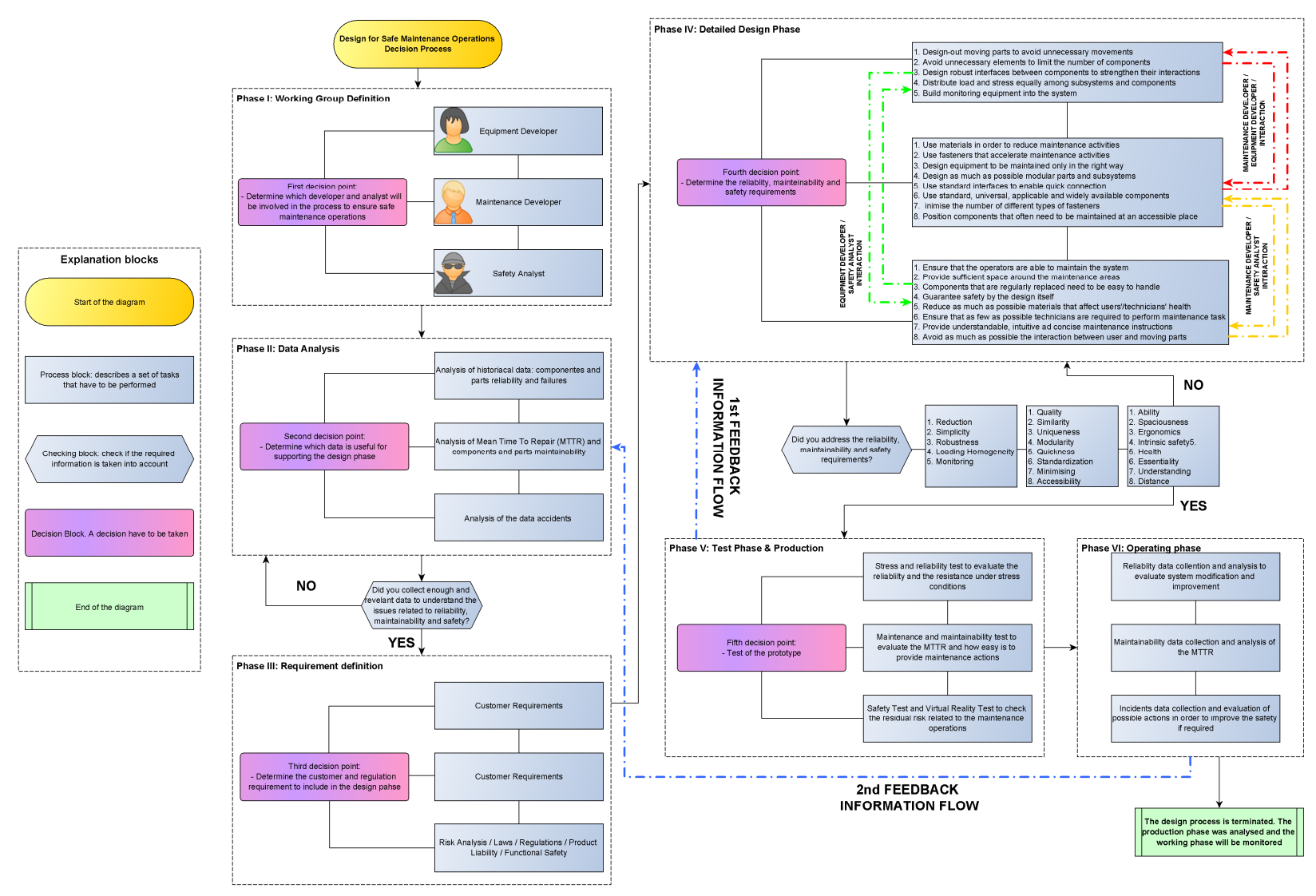

Fig. 6. Design for safe maintenance operations decision process

Table 5. Phases of the design for safe maintenance operations process

\begin{tabular}{ll}
\hline Phase & Characteristic \\
\hline Phase I: Working Group Definition & Equipment Developer, Maintenance Developer and Safety Analyst selection \\
Phase II: Data analysis & Gathering of the most useful information for the design \\
Phase III: Requirement Definition & Analysis of the requirements for the equipment \\
Phase IV: Detailed Design Phase & Design of the equipment \\
Phase V: Test and Production & Test and production of the equipment \\
Phase VI: Operating Phase & Use of the equipment \\
\hline
\end{tabular}

\section{Detailed Design Phase for Addressing Reliability, Maintainability and Safety: Phase IV}

With the gathering of the right data during the Phase II, it has to be considered the core and the most breakable step of the process due to the affection of the errors on the final result. The detailed design phase was implemented partially following the indications published by Mulder et al. (2012) in the "Design for maintenance - Guidelines to enhance maintainability, reliability and supportability of industrial products" (2012) and integrating them with the aspects related to the unavoidable design principles for ensuring safety.

As shown in Table 6, 5 principles for the reliability, 8 for the maintainability and 8 for the safety topics are identified and are stigmatized with one keyword. The authors tried to keep the amount of principles (and related keywords) as low as possible to emphasize only the essential aspects for a general design.

As said before, the best result in terms of embedded solutions will be reached only with a tight collaborations between equipment, maintenance developers and safety analyst to create solid and smooth interactions among the different principles. In this phase, the design process has to be shared as much as possible, to create a symbiosis within the reliability, maintainability and safety levels.

With the same purpose adopted for the Phase II, this Phase (IV) is followed by a checking block to verify if all the identified principles have been addressed during the design moments; the keywords related to the principles are extremely useful in order to visualise the main issues and they can play as final checklist before the test and production. 
1. Design-out moving parts to avoid unnecessary movements

2. Avoid unnecessary elements to limit the number of components

Reduction

3. Design robust interfaces between components to strengthen their interactions

Simplicity

4. Distribute load and stress equally among subsystems and components

5. Embed real-time sensors to control the machine

Principle - Maintainability

1. Use materials in order to reduce maintenance activities

2. Use tools that can make maintenance operations easier

3. Design equipment to be assembled and disassembled correctly

4. Design as much as possible modular parts and subsystems

5. Use standard interfaces to enable quick connection

6. Use standard, universal, applicable and widely available components

7. Minimise the number of different types of fasteners

8. Position components that often need to be maintained at an accessible place

Principle - Safety

1. Train the operators to correctly maintain the system

2. Design the system to have the proper space for operator's movements

3. Design the components to be easy to handle

4. Guarantee safety by the design itself

5. Reduce as much as possible materials that affect users'/technicians' health

6. Design system to be maintained with the few technicians

7. Write understandable, intuitive ad concise manuals

8. Avoid as much as possible the interaction between user and moving parts

Robustness

Loading Homogeneity

Monitoring

Keyword

Quality

Similarity

Uniqueness

Modularity

Quickness

Standardization

Minimising

Accessibility

Keyword

Ability

Spaciousness

Ergonomics

Intrinsic safety

Health

Essentiality

Understanding

Distance

\section{The Feedback Information Flow: Phase V, VI}

The Phase V and VI represent the Test/Production and the Operating moment. The production team has the opportunity to perform stress and reliability test in order to evaluate the equipment resistance under certain conditions, to assess how easy is to assemble and disassemble the equipment, checking MTTR having a first impression of the maintainability and to evaluate if the hazards identified have been removed or minimized providing a safe working conditions also for the maintenance operations. The introduction of Virtual Technologies can represent a remarkable improvement; in a virtual environment it is possible to let the worker free to make mistakes evaluating how the safety levels react to them in terms of accident prevention.

These actions give to the design team important feedback (1st Feedback Information Flow) for a second equipment improvements.

But, in the same way an important source of data and information is the Operating Phase (VI): the constant monitoring of an equipment in terms of failure rates, time dedicated to maintenance operations and in terms of accidents and occupational diseases repairing and replacing parts (2nd Feedback Information Flow) permits to collect valuable suggestions for modifications of the equipment during the design phase, increasing reliability and decreasing unexpected downtimes and residual risks related to the equipment.

\section{Reflections}

The proposed statistics of the accidents during the maintenance operations and the analysed event underline how the patterns of the injuries related to the moving parts present common characteristics. Although it is nearly impossible to completely eliminate them, the goal of a well-designed maintainability of the equipment from a human factor perspective is at least to minimize the impacts of the human errors.

But, as discussed in the previous paragraphs the preventive actions have to be put in place on two different levels: (i) on a system approach and (ii) on a person approach. The system approach lies on a more strategical and design level; considering the human fallible, it offers a more reliable safe barrier in every moment. For these reasons during such high-risk operations, as the maintenance is, the application of this approach covers a so vital role. The improvement of the maintainability of the machine to increase not only he technical performance but also the safety level is one of the challenge for during maintenance operations on the belt conveyors.

The system approach lies on a more strategical level; considering the human fallible, it offers a more reliable safe barrier in every moment. For these reasons during such high-risk operations, as the maintenance is, the application of this approach covers a so vital role.

On the other hand, with the person approach, constant formation, information and training should be offered to the worker to ensure the proper level of attention and the correct risk perception during the entire 
work shift to properly face the residual risks. As mentioned by Ouédraogo et al. (2011), usually we observe several risks perceptions to the same situations due to factors as fear, culture, education, society and experiences; it means that the training sessions should be worker-tailored to better fit in the previous own experiences and to transfer a significant message.

According to the analysis results, the only application of a fixed guard does not offer the request level of safety to the maintenance personnel. For instance, the interaction between user and moving parts, highlighted with the keyword "Distance" in the Design for Safe Maintenance Operations Decision Process is not respected during the maintenance operations since the workers was able to access to the pulley while it was working.

\section{Conclusion and Future Research}

In this paper, we back-analysed an accident occurred during the maintenance operations (replacement of a load carrying roller) of a conveyor belt in a crushing and milling plant. As consequence of the operation, the employee lost his arm.

According to the root causes obtained after the application of a post-event investigation protocol based on the combination of Cause-Consequence and Fault Tree Analysis, the responsibilities for the injury lie both:

- On a lack in the safety measures for the maintenance operations (no interlock guard was in place to mitigate the human fallibility) showing issues in the safety measures that means a poor identification of the possible hazards during the risk assessment process

- On a negligent behaviour of the employee due to low rates of involvement of the operators in OHS training among the SMEs, generating dangerous hazards misunderstandings during the operations

The prevention of accidents with dramatic consequences is of paramount importance for the efficiency of the system. Only a careful Risk Assessment and Management, combined with a detailed design project where reliability, maintainability and safety are seriously taken into account, could ensure safe working conditions on machines, equipment and plants for the maintenance operations.

The Design for Safe Maintenance Operations Decision Process starts from the very first step with the aim of merging together all the actions connected to technical performance of the equipment during the working phase (reliability), to the easiness to repair the equipment (maintainability) and to the risk that the operators have to face during the maintenance operations (safety).

The challenge for the further research is lying on the correct management of the Feedback Information Flows; collecting and gathering the information and providing the right data to the right point in the design and production process would be an outstanding achievement for improving the design characteristics of the equipment and the safety of the workers.

\section{Acknowledgement}

The presented paper was made achievable with the precious investigation data provided by the Environmental Inspectorate Office of the Turin Province, led by Dr. Paola Molina.

The authors also extend their words of gratitude to the reviewers for their invaluable help in improving this publication providing essential comments, suggestions and interesting references.

\section{Author's Contributions}

Alberto Martinetti: Contributed to prepare, develop and carry out the analysis of the presented injury and the design of the process for minimizing the risk during maintenance operations.

Leo A.M. van Dongen: Contributed to prepare, develop and carry out the design of the process for minimizing the risk during maintenance operations and the general maintenance aspects.

Raffaele Romano: Contributed to prepare, develop and carry out the statistical impact and typology of the injuries in the mining sector and the analysis of the presented injury.

\section{Ethics}

The corresponding author confirms that all of the other authors have read and approved the manuscript and no ethical issues involved.

\section{References}

Anderson, F.A., 1995. OSHA Regulations and their implications. Safety in the Underground Construction and Operation of the Exploratory Studies Facility at Yucca Mountain, Committee on Tunneling Technology, National Research Council, National Academies Press, pp: 23-31.

Bagherpour, R., R. Yarahmadi and A. Khademian, 2015. Safety Risk Assessment of Iran's underground coal mines based on preventive and preparative measures. Human Ecological Risk Assessment: An Int. J., 21: 2223-2238.

DOI: $10.1080 / 10807039.2015 .1046418$

Blaise J.C., P. Lhoste and J. Cicotelli, 2003. Formalisation of normative knowledge for safe design. Safety Sci., 41: 241-261. DOI: $10.1016 / \mathrm{S} 0925-7535(02) 00004-8$ 
Blaise, J.C., E. Levrat and B. Iung, 2014. Process approach-based methodology for safe maintenance operation: From concepts to SPRIMI software prototype. Safety Science, 70: 99-113.

DOI: $10.1016 /$ j.ssci.2014.05.008

Blanchard, B. and W.J. Fabrycky, 2014. Systems Engineering and Analysis, 5th Edn., Boston : Prentice Hall, pp: 786. ISBN-13: 9780132217354

Bonafede, M., M. Corfiati, D. Gagliardi, F. Boccuni and M. Ronchetti et al., 2016. OHS management and employers' perception: Differences by firm size. Safety Sci., 89: 11-18.

DOI: $10.1016 /$ j.ssci.2016.05.012 0925-7535

British Standards Institution, 2007. OHSAS 18001:2007 concerning Occupational Health and Safety (OHS) Management systems-Requirements.

Center for Chemical Process Safety of the American Institute of Chemical Engineers, 1992. Guidelines for Hazard Evaluation Procedures, 2nd Edn., American Institute of Chemical Engineers, New York, USA.

Chinniah, Y., 2015. Analysis and prevention of serious and fatal accidents related to moving parts of machinery. Safety Sci., 75: 163-173.

DOI: $10.1016 /$ j.ssci.2015.02.004

Council of the European Communities, 1989. Council Directive EEC 89/391 concerning the introduction of measures to encourage improvements in the Safety and Health of workers at work.

Demichela, M., L. Monai and M. Patrucco, 2011. The Deep Analysis of the Accidents as an Essential Prevention Tool: A Post-Event Investigation Protocol for the Analyst (in Italian), In: Ingegneria Forense: Metodologie, Protocolli e casi di Studio, Chiaia, B. and D. Flaccovio (Eds.), Palermo, Italy, pp: 231-246.

European Agency for Safety and Health at Work, 2010. Maintenance and OSH - A statistical picture. Facts 90, Belgium.

European Committee for the Standardization, 1997. European Standard EN 953 + A1:2009 concerning Safety of machinery - Guards - General requirements for the design and construction of fixed and movable guards.

European Committee for the Standardization, 2008. European Standard (ISO) 13857 concerning Safety of machinery - Safety distances to prevent hazard zones being reached by upper and lower limbs.

European Committee for the Standardization, 2010. European Standard EN 741 + A1:2010 concerning Continuous handling equipment and systems Safety requirements for systems and their components for pneumatic handling of bulk material.

European Committee for the Standardization, 2010. European Standard (ISO) 12100 concerning new standard for guard interlocking devices.
European Committee for the Standardization, 2010. European Standard 13306 concerning maintenance maintenance terminology.

European Community, 2006. European Directive 2006/42 concerning the machinery.

European Statistics on Accidents at Work (ESAW), 2015.

Groves, W., V. Kecojevic, D. Komljenovic and M. Radomsky, 2007. An analysis of equipmentrelated fatal accidents in U.S. mining operations: 1995-2005. Safety Sci., 45: 864-874. DOI: $10.1016 /$ j.ssci.2006.08.024

Hasle, P., P. Kines and L.P. Andersen, 2009. Small enterprise owners' accident causation attribution and prevention, Safety Science, 47: 9-19. 10.1016/j.ssci.2007.12.005

Horberry, T., R. Burgess-Limerick and L.J. Steiner, 2010. Human factors for the design, operation and maintenance of mining equipment, CRC Press.

Kontogiannis, T., V. Leopoulos and N. Marmaras, 2000. A comparison of accident analysis techniques for safety-critical man-machine systems. Int. J. Industrial Ergonomics, 25: 327-347. DOI: 10.1016/S0169-8141(99)00022-0

Lind, S. and S. Nenonen, 2008. Occupational risks in industrial maintenance. J. Quality Maintenance Eng., 14: 194-204. DOI: $10.1108 / 13552510810877683$

Luzzi, R., L. Maida, A. Martinetti and M. Patrucco, 2013. Information, formation and training for the maintenance operations: The lesson learned from fatal accidents. Chemical Eng. Trans., 32: 229-234. DOI: $10.3303 /$ CET1332039

Lynas, D. and T. Horberry, 2011. Human factor issues with automated mining equipment. Ergonomics Open J., 4: 74-80.

Mine Safety and Health Administration (MSHA) 30 CFR § 56/57.14107. Program Policy Manual Volume IV, Metal and Nonmetal Mines, Interpretation, Application and Guidelines on Enforcement of 30 CFR.

Mine Safety and Health Administration (MSHA), 2010. Guarding conveyor belts at metal and nonmetal mines.

Mulder, W., J. Blok, S. Hoekstra and F.G.M. Kokkeler, 2012. Design for maintenance. Guidelines to enhance maintainability, reliability and supportability of industrial products. Enschede, University of Twente.

OSHC Quebec, 2003. Safety of belt conveyors: Guide of the use. National Library of Quebec.

Ouédraogo, A., A. Groso and T. Meyer, 2011. Risk analysis in research environment - Part I: modeling lab criticity index using improved risk priority number. Safety Science, 49: 778-784.

DOI: $10.1016 /$ j.ssci.2011.02.006 
Reason, J., 2000. Human error: Models and management. BMJ, 320: 768-770.

DOI: $10.1136 / \mathrm{bmj} .320 .7237 .768$

Ruff, T., P. Coleman and L. Martini, 2011. Machinerelated injuries in the US mining industry and priorities for safety research. Int. J. Injury Control Safety Promotion, 18: 11-20.

DOI: $10.1080 / 17457300.2010 .487154$.

Sbarzaglia, D. and V. Correggia, 2012. Crushing and milling plants: Considerations on the new safety and health regulations. Marmomacc - Verona.

Tappura, S., S. Sirpa Syvänen and K. Leena Saarela, 2014. Challenges and needs for support in managing Occupational Health and Safety from managers' viewpoints. Nordic J. Working Life Studies, 4: 31-51. DOI: $10.19154 /$ njwls.v4i3.4178
Vanaker, T. and T. van Diepen, 2016. Design Support for Maintenance Tasks using TRIZ. Procedia CIRP, 39: 67-72. DOI: 10.1016/j.procir.2016.01.167

Vatn, J. and T. Aven, 2010. An approach to maintenance optimization where safety issues are important. Reliability Engineering System Safety, 95: 58-63. DOI: 10.1016/j.ress.2009.06.002

Väyrynen, S., K. Häkkinen and T. Niskanen, 2014. Integrated Occupational Safety and Health Management: Solutions and Industrial Cases. 1st Edn., Springer, pp: 306. ISBN-10: 331913180X 